\title{
Acinetobacter baumannii infection in a medical intensive care unit: The impact of strict infection control
}

\author{
A M Aboshakwa, MD, FCP (SA), MMed (Int); U Lalla, MB ChB, FCP (SA), MMed (Int), Cert Crit Care (SA); \\ E M Irusen, MB ChB, FCP (SA), PhD; C F N Koegelenberg, MB ChB, MMed (Int), FCP (SA), FRCP (UK), Cert Pulm (SA), PhD \\ Division of Pulmonology, Department of Medicine, Stellenbosch University and Tygerberg Academic Hospital, Cape Town, South Africa \\ Corresponding author: A M Aboshakwa(e_aboshakwa@yahoo.com)
}

\begin{abstract}
Background. Acinetobacter baumannii is a waterborne organism that preferentially colonises aquatic environments. Infections usually involve organ systems that have a high fluid content. Multidrug-resistant (MDR) A. baumannii is recognised to be among the most difficult antimicrobial-resistant Gram-negative bacilli to prevent and treat in the nosocomial setting.

Objective. To determine the utility of concomitant implementation of a strict antimicrobial stewardship programme and comprehensive infection control measures to control MDR A. baumannii in a medical intensive care unit (ICU).

Methods. We retrospectively compared the relative incidence of A. baumannii infections in our unit over a 1-year period before (2012) and after (2016) the implementation of strict infection control bundles. Patients with A. baumannii infections were identified using the microbiology database of the National Health Laboratory Service's central data warehouse. The total number of admissions and clinical data were derived from the ICU registry.

Results. A. baumannii was isolated from 43/263 patients (16.35\%) in 2012 compared with $37 / 348$ patients in 2016 (10.63\%, $p=0.03$; relative risk reduction $=35 \%$ ). We found almost $100 \%$ sensitivity to colistin and tigecycline, but $90 \%$ resistance to carbapenem antibiotics.

Conclusion. The introduction of strict infection control bundles had a statistically significant and clinically meaningful impact on the incidence of nosocomial A. baumannii infection in the medical ICU.
\end{abstract}

Afr J Thoracic Crit Care Med 2019;25(1):10-13. DOI:10.7196/AJTCCM.2019.v25i1.239

Acinetobacter baumannii is a pleomorphic aerobic Gram-negative bacillus that is commonly isolated from the hospital environment and hospitalised patients. ${ }^{[1]}$ It is a waterborne organism that preferentially colonises aquatic environments and, in hospitalised patients, is often cultured from sputum or respiratory secretions, wounds, urine, and irrigating and intravenous solutions. ${ }^{[2]}$

A. baumannii has a low virulence but can cause infection in patients with organ transplants and febrile neutropenia. Most A. baumannii isolates recovered from hospitalised patients, particularly those recovered from respiratory secretions and urine, represent colonisation rather than infection; however, care must be exercised in making that determination. Multiple factors increase the risk for acquiring an A. baumannii infection in the hospital setting, such as prior antibiotic exposure, intensive care unit (ICU) admission, use of a central venous catheter, mechanical ventilation or haemodialysis. ${ }^{[2,3]}$

A. baumannii infections usually involve organ systems that have a high fluid content (i.e. the respiratory and urinary tracts, cerebrospinal fluid and peritoneal fluid). These infections may occur as outbreaks rather than isolated cases of nosocomial infection. Infections may also complicate continuous ambulatory peritoneal dialysis or cause catheter-associated bacteriuria. ${ }^{[4]}$

Multidrug-resistant (MDR) A. baumannii is recognised to be among the most difficult antimicrobial-resistant Gram-negative bacilli to prevent and treat. Increasing antimicrobial resistance among A. baumannii isolates has been documented, although definitions vary in the literature; the most widely used definition of MDR A. baumannii is resistance to more than three classes of antibiotics. ${ }^{[5]}$ Antimicrobial resistance greatly limits the therapeutic options for patients who are infected with this organism, especially if isolates are resistant to the carbapenem antibiotics. Therapeutic options for the treatment of MDR A. baumannii infection are thus limited. The development or discovery of new therapies, well-controlled clinical trials of existing antimicrobial regimens and combinations, and greater emphasis on the prevention of healthcare-associated transmission of MDR A. baumannii infection are essential. ${ }^{[6]}$

Only a few studies have been performed to assess A. baumannii prevalence and resistance in the ICU setting. National awareness of infection control and judicious antimicrobial use is required to overcome this burden.$^{[7]}$ The US Institute for Healthcare Improvement has developed the concept of 'bundle' implementation in healthcare to facilitate the clinician's ability to deliver bedside care more reliably and effectively. ${ }^{[8]}$

A 'bundle' is a group of evidence-based care components for a given disease, which, when executed together, may result in better outcomes than if implemented individually. ${ }^{[8]}$ Concomitant implementation of strict antimicrobial stewardship programmes and comprehensive infection control measures have contributed to controlling endemic MDR A. baumannii effectively in the ICU setting. ${ }^{[9]}$

Bundles, including those for central line-associated bloodstream infection (CLABSI), ventilator-associated pneumonia (VAP) and 
catheter-associated urinary tract infection (CAUTI), were introduced at Tygerberg Academic Hospital in 2013. In addition, an antibiotic stewardship programme commenced in the same year.

The primary objective of this study was to compare the relative incidence of A. baumannii in the adult medical ICU of Tygerberg Academic Hospital before and after the introduction of the various bundles. The secondary aim was to determine the antimicrobial susceptibility of A. baumannii during these study periods.

\section{Methods}

Study description and population

In this retrospective and analytical study, we compared the relative incidence of positive A. baumannii culture(s) in two 1-year periods (2012 and 2016) in the medical ICU of Tygerberg Academic Hospital. Patients with A. baumannii isolates were identified using the microbiology database of the National Health Laboratory Service's central data warehouse. In addition, the ICU registry was used to determine the total number of ICU admissions for 2012 and 2016. All patients older than 13 years from whom A. baumannii was isolated (from any site) were included. Patients younger than 13 years and those with incomplete patient data were excluded. Ethical approval for this retrospective analysis was granted by the Stellenbosch University Research Ethics Committee. This approval included a waiver of consent owing to the retrospective nature of this study.

Bundles for CLABSI and VAP were introduced in our unit in 2013. Personnel completed a training programme that included bedside training and didactic lectures on the theory and implementation of the bundles (including the introduction of checklists). Bundle compliance was assessed by trained staff four times per day (according to further checklists) and reported monthly. The bundles complemented the existing antibiotic stewardship interventions, which included antibiotic authorisation and weekly ICU rounds by infectious disease specialists.

\section{Data collection and processing}

The source of A. baumannii isolates (sputa, blood, tissues, body fluid and catheters) during admission to our unit and antimicrobial susceptibility patterns were captured. Data obtained included patient demographics, comorbidities, length of stay, admission diagnosis and outcome. The relative incidence over the study period was calculated as the number of patients with A. baumannii isolates for a specific year relative to the total number of admissions for that year. No patients were routinely screened for A. baumannii during the two periods investigated.

\section{Statistical analysis}

Demographic data with a normal distribution, such as age, gender and race, are reported as means with standard deviations (SD). Data that were not normally distributed are reported as medians with interquartile ranges. Fisher's exact test was used for categorical variables. Statistical significance was regarded as $p<0.05$ unless otherwise stated. Continuous variables are presented as mean (SD).

\section{Results}

A total of 263 patients (115 male) were admitted in 2012. This group had a mean age of 39.7 (15.0) years and a mean APACHE score of
15.4 (9.3). In 2016, a total of 348 patients ( $n=142$ male) were admitted, with a mean (SD) age of 39.2 (16.1) years and a mean APACHE score of $18.6(10.2)$.

A. baumannii was isolated from $43 / 263$ patients (16.35\%) in 2012 , and $37 / 348$ patients in $2016(10.63 \%, p=0.03$; relative risk reduction $=35 \%$ ). Bundles had to be observed in 14 patients to prevent one infection.

The sources of the positive cultures are summarised in Fig. 1 and Fig. 2 for 2012 and 2016, respectively. The tracheal aspirate was a major source in both years, with a rate of $70 \%$ in 2012 and 33\% in 2016.

Antibiotic sensitivities for both 2012 and 2016 are summarised in Fig. 3. In general, A. baumannii isolates were almost $100 \%$ sensitive to colistin and tigecycline, but only $10 \%$ were sensitive to carbapenems.

The mean length of hospital stay was 12.9 (10.9) days in 2012 compared with 10.2 (9.5) days in 2016. In both years, the most common admission diagnosis in patients who went on to develop an A. baumannii infection was that of community-acquired pneumonia. The mortality rate of patients with A. baumannii infection was $27.9 \%$ $(12 / 43)$ in 2012 and $18.9 \%(7 / 37)$ in 2016.

\section{Discussion}

The introduction of strict infection control bundles had a statistically significant and clinically meaningful impact on the incidence of nosocomial A. baumannii infection in the medical ICU. We observed a $5.7 \%$ decrease in the isolation of A. baumannii following the conscientious introduction of these bundles.

Our observations are comparable to published data. An Italian study showed a significant reduction in the spread of MDR A. baumannii among patients during an outbreak. ${ }^{[10]}$ A study that investigated the impact of bundles to reduce sepsis in the ICU showed a significant reduction in the rate of central catheter-related bloodstream infection. ${ }^{[11]}$ Similarly, decreased rates of CAUTI and VAP have been reported in the ICU after the implementation of the respective bundles. ${ }^{[12,13]}$ Overall, the implementation of the various bundles appears to have contributed significantly to reducing the incidence of sepsis in the ICU, especially with regard to resistant bacteria such as A. baumannii, and helps to decrease the spread of infection among ICU patients. ${ }^{[1,15]}$

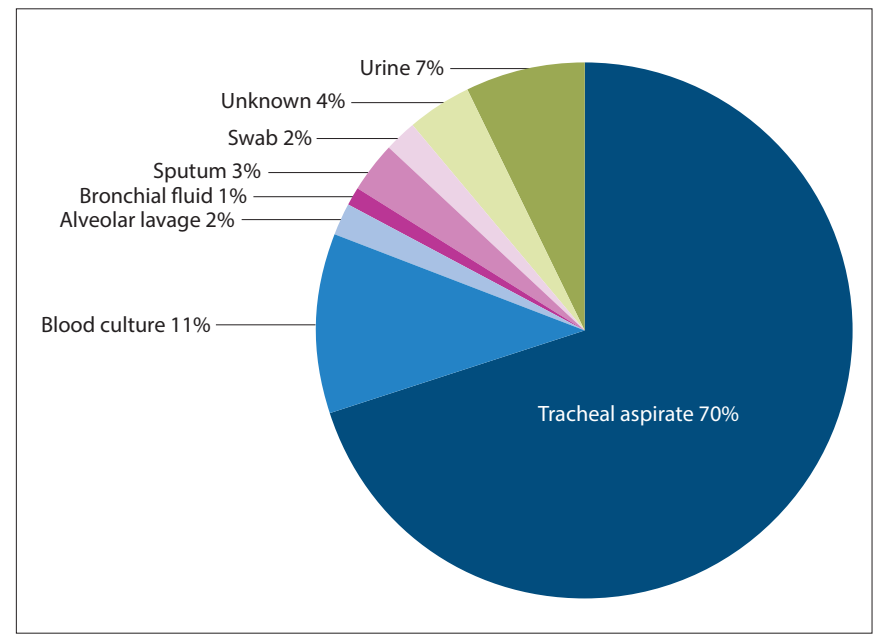

Fig. 1. Sources of positive cultures in 2012. 


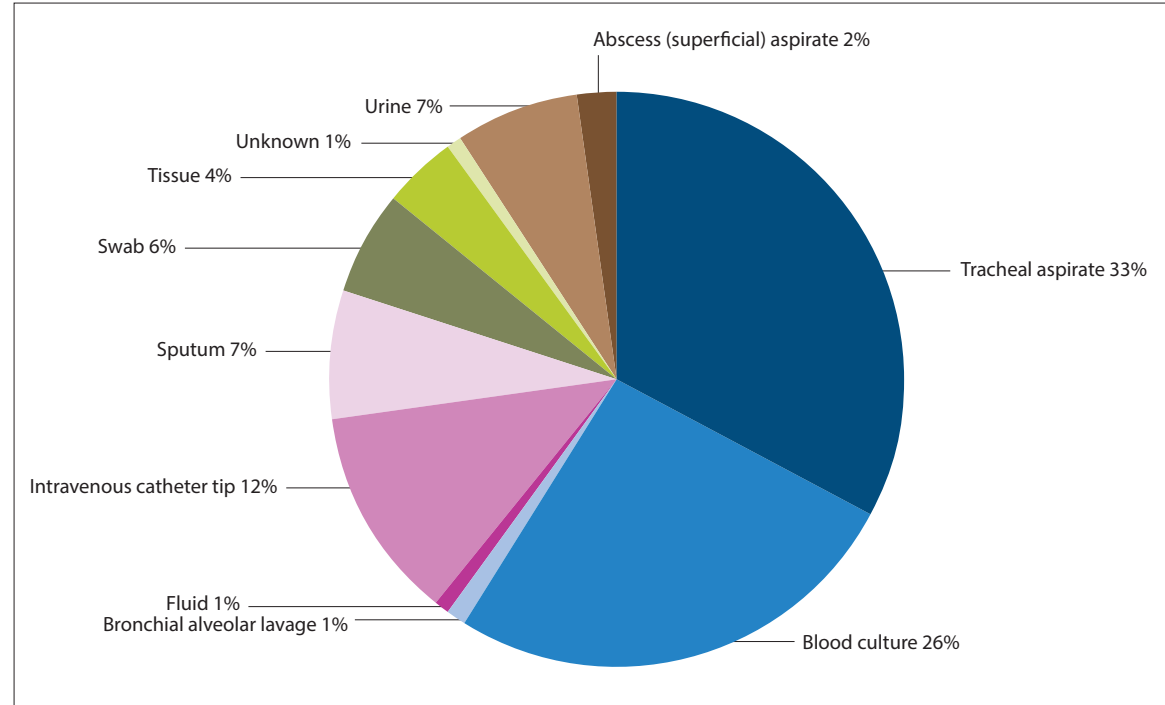

Fig. 2. Sources of positive cultures in 2016.

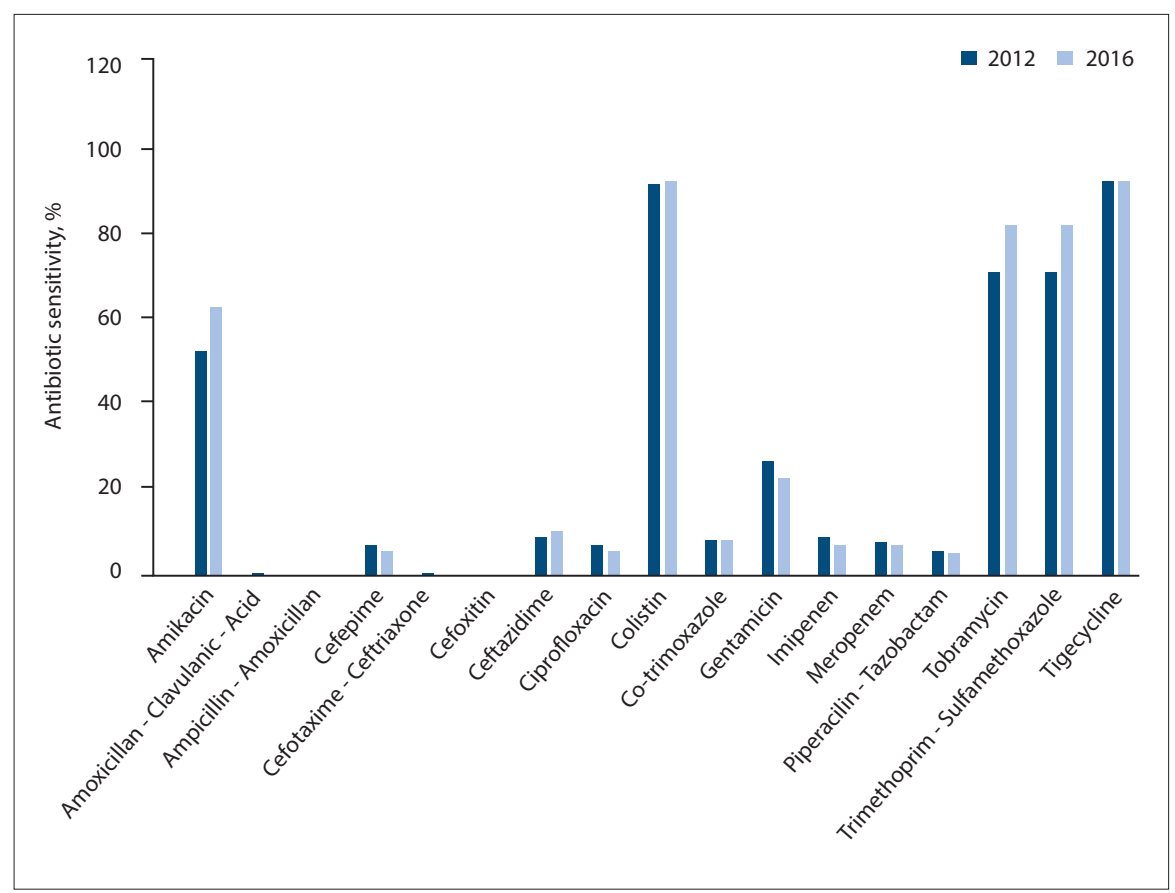

Fig. 3. Antibiotic resistance of Acinetobacter baumannii cultures in 2012 and 2016.

Although the incidence of $A$. baumannii infection is on the rise in developed countries, there is a paucity of data from Africa. ${ }^{[16-19]}$ We found mortality to be as high as $28 \%$ in 2012 and $19 \%$ in 2016. These figures are comparable to those reported by Trottier et al., ${ }^{[20]}$ who noted a mortality rate of $26.2 \%$ in critically ill American patients. Other investigators reported mortality rates of $8 \%-43 \%$ in developed countries and $33 \%-45 \%$ in developing countries. ${ }^{[21-23]}$ However, studies generally did not control for confounding risk factors such as age, disease severity and comorbidities. We biosynthesis. ${ }^{[26]}$ Colistin resistance is therefore still considered sporadic. Our study also confirmed an increase in carbapenem resistance. The mechanisms of development of carbapenem resistance in Acinetobacter spp. have been attributed to efflux pumps and outer membrane proteins. ${ }^{[27]}$ A. baumannii resistance to tigecycline may also develop via the acquisition of efflux pumps. ${ }^{[28]}$

The main strength of our study is that it was performed in a medical ICU with a low staff turnover and a workload that allowed for the strict implementation of the various bundles. Moreover, our policy of replacing central lines (1) in the case of a possible breach in sterility or (2) if a patient was transferred from another hospital may have impacted on the reduction in the rate of CLABSI seen in our unit.

\section{Study limitations}

Most of our ICU patients were referred from secondary hospitals within the drainage area of Tygerberg Academic Hospital. Patients were also intubated, which is known to increase the rate of A. baumannii colonisation or infection, independent of the measures implemented. Owing to the wide drainage area, it remains unclear whether patients were colonised or infected with A. baumannii prior to their ICU admission at our institution, as no routine screening was performed. Another potential limitation is that we could report the incidence of $A$. baumannii relative only to total ICU admissions during a period and not to the total population at risk. In addition, we could not report on the clinical significance of the positive isolates and whether treatment was instituted or not.

\section{Conclusion}

Despite some limitations, we were able to show that the introduction of strict infection control bundles had a significant and clinically meaningful impact on the incidence of nosocomial A. baumannii infections in the medical ICU setting. therefore likely to transcend resource and economic boundaries and should include strict attention to infection control and stringent antibiotic stewardship.

Cases of A. baumannii resistance to colistin have been reported in a few studies. Such resistance can be acquired through mutations in the $l p x A, l p x C$ and $l p x D$ genes, leading to complete loss of lipopolysaccharide
Acknowledgements. We thank Dr Riezaah Abrahams from Infection Prevention and Control for his assistance in collecting data for this project. initiated the study, analysed the data and wrote the manuscript. All authors contributed to the
Author contributions. CFNK, UL and AMA 
study design, data collection and editing of the manuscript. EMI critically reviewed the data analysis and edited the final draft of the manuscript.

Funding. None.

Conflicts of interest. None.

1. Ntusi NB, Badri M, Khalfey H, et al. ICU-associated Acinetobacter baumannii colonisation/infection in a high HIV-prevalence resource-poor setting. PLoS One 2012;7:e52452. https://doi.org/10.1371/journal.pone.0052452

2. Nguyen MH, Harris SP, Muder RR, Pasculle AW. Antibiotic-resistant Acinetobacter meningitis in neurosurgical patients. Neurosurgery 1994;35(5):851-855. https://doi. org/10.1097/00006123-199411000-00008

3. Krol V, Hamid NS, Cunha BA. Neurosurgically related nosocomial Acinetobacter baumannii meningitis: Report of two cases and literature review. J Hosp Infect 2009;71(2):176-180. https://doi.org/10.1016/j.jhin.2008.09.018

4. Alberti C, Brun-Buisson C, Burchardi H, et al. Epidemiology of sepsis and infection in ICU patients from an international multicentre cohort study. Intensive Care Med 2002;28(2):108-121. https://doi.org/10.1007/s00134-001-1143-Z

5. Manchanda V, Sanchaita S, Singh NP. Multidrug resistant Acinetobacter. J Glob Infect Dis 2010;2(3):291-304. https://doi.org/10.4103/0974-777X.68538

6. Viehman JA, Nguyen M-H, Doi Y. Treatment options for carbapenem-resistant and extensively drug-resistant Acinetobacter baumannii infections. Drugs 2014;74(12):1315-1333. https://doi.org/10.1007/s40265-014-0267-8

7. Uwingabiye J, Frikh M, Lemnouer A, et al. Acinetobacter infections prevalence and frequency of the antibiotics resistance: Comparative study of intensive care units versus other hospital units. Pan Afr Med J 2016;23:191. https://doi.org/10.11604/ pamj.2016.23.191.7915

8. Horner DL, Bellamy MC. Care bundles in intensive care. Contin Educ Anaesth Crit Care Pain 2012;12(4):199-202. https://doi.org/10.1093/bjaceaccp/mks021

9. Cheon S, Kim M-J, Yun S-J, Moon JY, Kim Y-S. Controlling endemic multidrugresistant Acinetobacter baumannii in intensive care units using antimicrobial stewardship and infection control. Korean J Intern Med 2016;31(2):367-374. https:// doi.org/10.3904/kjim.2015.178

10. Bianco A, Quirino A, Giordano M, et al. Control of carbapenem-resistant Acinetobacter baumannii outbreak in an intensive care unit of a teaching hospital in Southern Italy. BMC Infect Dis 2016;16:747. https://doi.org/10.1186/s12879-016-2036-7

11. Padilla Fortunatti CF. Impact of two bundles on central catheter-related bloodstream infection in critically ill patients. Rev Lat Am Enfermagem 2017;25:e2951. https://doi. org/10.1590/1518-8345.2190.2951

12. Lai CC, Lee CM, Chiang HT, et al. Implementation of a national bundle care program to reduce catheter-associated urinary tract infection in high-risk units of hospitals in Taiwan. J Microbiol Immunol Infect 2017;50(4):464-470. https://doi.org/10.1016/j. jmii.2017.01.006

13. Eom JS, Lee MS, Chun HK, et al. The impact of a ventilator bundle on preventing ventilator-associated pneumonia: A multicenter study. Am J Infect Control 2014;42:34-37. https://doi.org/10.1016/j.ajic.2013.06.023

14. Teles F, Rodrigues WG, Alves MGTC, et al. Impact of a sepsis bundle in wards of a tertiary hospital. J Intensive Care 2017;5:45. https://doi.org/10.1186/s40560-017$0231-2$
15. Lee H, Lee H. Clinical and economic evaluation of multidrug-resistant Acinetobacter baumannii colonization in the intensive care unit. Infect Chemother 2016;48(3):174180. https://doi.org/10.3947/ic.2016.48.3.174

16. Zapor MJ, Moran KA. Infectious diseases during wartime. Curr Opin Infect Dis 2005;18(5):395-399. https://doi.org/10.1097/01.qco.0000182102.50430.2c

17. Vincent J, Bihari DJ, Suter PM, et al. The prevalence of nosocomial infection in intensive care units in Europe. Results of the European Prevalence of Infection in Intensive Care (EPIC) study. JAMA 1995;274(8):639-644. https://doi.org/10.1001/ jama.1995.03530080055041

18. Paul M, Weinberger M, Siegman-Igra Y, et al. Acinetobacter baumannii: Emergence and spread in Israeli hospitals 1997-2002. J Hosp Infect 2005;60:256-260. doi. org/10.1016/j.jhin.2005.01.007

19. Diekema DJ, Pfaller MA, Jones RN, et al. Trends in antimicrobial susceptibility of bacterial pathogens isolated from patients with bloodstream infection in the USA, Canada and Latin America. Int J Antimicrob Agents 2000;13:257-271. https://doi. org/10.1016/s0924-8579(99)00131-4

20. Trottier V, Namias N, Pust DG, et al. Outcomes of Acinetobacter baumannii infection in critically ill surgical patients. Surg Infect 2007;(8):437-443. doi.org/10.1089/ sur.2006.029

21. Sunenshine RH, Wright M-O, Maragakis LL, et al. Multidrug-resistant Acinetobacter infection mortality rate and length of hospitalization. Emerg Infect Dis 2007;13(1):97103. https://doi.org/10.3201/eid1301.060716

22. Daniels TL, Deppen S, Arbogast PG, Griffin MR, Schaffner W, Talbot TR. Mortality rates associated with multidrug-resistant Acinetobacter baumannii infection in surgical intensive care units. Infect Control Hosp Epidemiol 2008;29(11):1080-1083. https://doi.org/10.1086/591456

23. Falagas ME, Bliziotis IA, Siempos II. Attributable mortality of Acinetobacter baumannii infections in critically ill patients: A systematic review of matched cohort and case-control studies. Crit Care 2006;10(2):R48. https://doi.org/10.1186/cc4869

24. Agarwal R, Gupta D, Ray P, Aggarwal AN, Jindal SK. Epidemiology, risk factors and outcome of nosocomial infections in a respiratory intensive care unit in North India. J Infect 2006;53:98-105. https://doi.org/10.1016/j.jinf.2005.10.021

25. Chen CH, Lin LC, Chang YJ, Huang CC, Liu CE, Young TG. Analysis of prognostic factors in 95 patients with Acinetobacter baumannii bacteremia. Infection 2003;31:331335. https://doi.org/10.1007/s15010-003-3223-1

26. Carretero-Ledesma M, García-Quintanilla M, Martín-Peña R, Pulido MR, Pachó J, McConnell MJ. Phenotypic changes associated with colistin resistance due to lipopolysaccharide loss in Acinetobacter baumannii. Virulence 2018;9(1):930-942. https://doi.org/10.1080/21505594.2018.1460187

27. Zhang Y, LiZ, He X, et al. Overproduction of efflux pumps caused reduced susceptibility to carbapenem under consecutive imipenem-selected stress in Acinetobacter baumannii. Infect Drug Resist 2017;11:457-467. https://doi.org/10.2147/idr.s151423

28. Deng M, Zhu M-H, Li J-J, et al. Molecular epidemiology and mechanisms of tigecycline resistance in clinical isolates of Acinetobacter baumannii from a Chinese university hospital. Antimicrob Agents Chemother 2014;58(1):297-303. https://doi. org/10.1128/aac.01727-13

Accepted 14 March 2019 